\title{
Receiver Design in Molecular Communications: An Approach Based on Artificial Neural Networks
}

\author{
Xuewen Qian \\ Paris-Saclay University \\ Laboratory of Signals and Systems (L2S) \\ CNRS - CentraleSupélec - Université Paris Sud \\ Paris, France \\ qian.xuewen@12s.centralesupelec.fr
}

\author{
Marco Di Renzo \\ Paris-Saclay University \\ Laboratory of Signals and Systems (L2S) \\ CNRS - CentraleSupélec - Université Paris Sud \\ Paris, France \\ marco.direnzo@12s.centralesupelec.fr
}

\begin{abstract}
The design of communication systems typically relies on the development of mathematical models that describe the underlying communication channel. In many communication systems, however, accurate channel models may not be known, or the models may not be accurate enough or even not available for efficient system design. In these scenarios, a completely new approach to communication system design and analysis is required. An example of such situations arises in the emerging research field of molecular communications, for which it is very difficult to develop accurate analytical models for several operating scenarios. In this context, the use of data-driven techniques based on artificial neural networks may provide an alternative and suitable solution towards the design and analysis of molecular communication systems. In this paper, we explore the potential of artificial neural networks for application to the design of robust receiver schemes. We study a molecular communication system in the presence of inter-symbol interference and show that a receiver based on artificial neural networks can be trained by using only empirical (raw) data and can provide the same performance as a receiver that has perfect knowledge of the underlaying channel model.
\end{abstract}

Index Terms-Molecular communications, artificial neural networks, receiver design and optimization.

\section{INTRODUCTION}

At the nano-scale, traditional electromagnetic-based transmission techniques may not be suitable to enable nano-devices to communicate [1]. An alternative option is to use an emerging paradigm that is known as Molecular Communication (MC) [2], where the information is transmitted by using chemical signals instead of radio-frequency signals.

Similar to radio communication systems, a MC system is made of a transmitter, a receiver, and the transmission channel. The information is transmitted via the release of information particles, e.g., by encoding the data into the number of particles that are released, the type of particles that are released, or the release time of the particles [2]. If the information is encoded into the number of particles that are released, the corresponding modulation scheme is referred to as Concentration Shift Keying (CSK) modulation.

In the context of MC systems, diffusion is the easiest option to allow information particles propagating from the transmitter to the receiver. Due to the intrinsic characteristics of diffusion, the resulting transmission channel is usually affected by a non- negligible Inter-Symbol Interference (ISI), which, if not taken into account for system optimization, may severely degrade the system performance [3], [4]. For this reason, we focus our attention on optimizing MC systems in the presence of ISI.

In MC systems, a simple approach to demodulate the transmitted binary symbols is to compare the number of received particles, $r_{i}$, with a fixed threshold, $\tau$, [5]. If $r_{i} \geq \tau$, the symbol is detected as 1 , otherwise it is detected as 0 . The threshold, $\tau$, of this threshold-based detector is relatively simple to be optimized in the absence of ISI or if the ISI is negligible. In general, on the other hand, $\tau$ needs to be optimized by taking the ISI into account in order to minimize the error probability and obtain good communication performance. In [6], the authors have proposed a scheme that uses the number of particles received in the previous time-slot, i.e., $r_{i-1}$, as the detection threshold in a given time-slot. An improved scheme has been proposed in [7], where a detection scheme that combines channel estimation and decision-feedback equalization is used. Other improved detection schemes have recently been proposed in [8] and [9].

The aforementioned approaches rely on the knowledge of the channel and system models. This, however, may not always be possible either due to the complexity of modeling the entire system in an accurate manner or due to the complexity of optimizing the resulting system model. In order to solve these issues, the authors of [10] have recently proposed a sequence detection scheme based on sliding bidirectional recurrent neural networks. With the aid of deep learning methods, the authors have shown that the scheme is capable of automatically learning the whole system from empirical data and of performing data detection without using complex channel estimation and data equalization techniques.

In the present paper, motivated by the promising results obtained in [10], we study the potential of applying Artificial Neural Network (ANN) methods for data detection of MC systems in the presence of ISI. Our study shows that ANNs without prior knowledge of the system model are capable of providing the same performance as conventional detection methods that rely on the perfect knowledge of the underlaying system model.

The reminder of this paper is organized as follows. In 


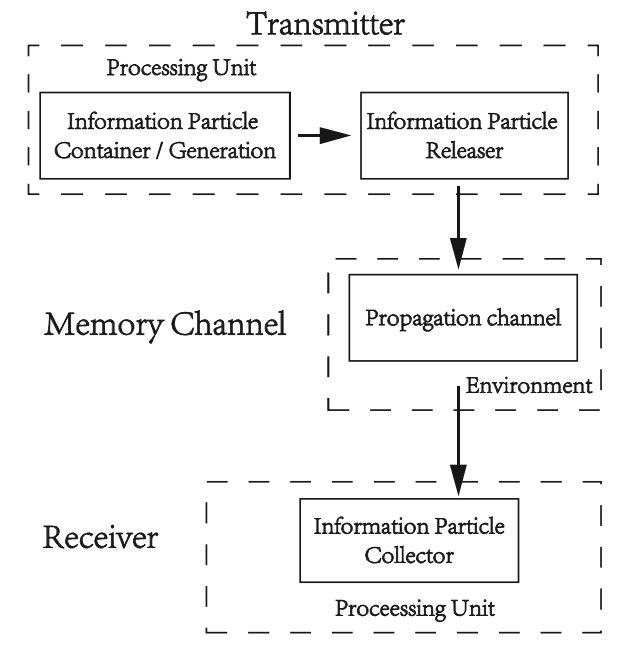

Fig. 1. Block diagram of a MC system.

Section II, we introduce the system model. In Section III, we study and optimize a threshold-based detector by relying upon "model-based" and "data-driven" methods. In Section IV, some simulation results are illustrated, which confirm that similar performance is obtained by using the two methods. Finally, Section V concludes the paper.

\section{System Model}

A MC system can be split in three parts: the transmitter, the channel, and the receiver. Figure 1 depicts the main components of a MC system. The transmitter generates or stores the information particles, which are released into the channel by a releaser. We assume that the transmitter is small enough to be modeled as a point. We assume that the MC system is deployed in a gaseous or aqueous environment, like the human body. We assume that the information particles propagate through the channel by means of Brownian motion. In particular, the particles diffuse randomly through the medium and the motion of a particle is independent of the motion of any other particles. Usually, a large number of information particles are emitted through the channel but not all of them reach the receiver in the considered time-slot. Some information particles remain in the channel and reach the receiver in subsequent time-slots: this causes the ISI. If not appropriately taken into account, the ISI may severely degrade the performance of $\mathrm{MC}$ systems. As an example, we consider an absorbing-type receiver [11].

In the considered system model, the transmitter is modeled as a point and the receiver is assumed not to be too close to it. An ultra large three-dimensional aqueous propagation medium without drift is considered. By assuming the transmitter to be located at $\boldsymbol{a}=(0,0,0)$ and the receiver at $\boldsymbol{b}=\left(b_{x}, b_{y}, b_{z}\right)$, the hitting rate of each information particle can be expressed as follows [6]:

$$
f_{\text {hit }}^{3 D}(t)=\frac{r(d-r)}{d \sqrt{4 \pi D t^{3}}} e^{-\frac{(d-r)^{2}}{4 D t}}
$$

where $\|\boldsymbol{a}-\boldsymbol{b}\|=d$ is the distance between transmitter and receiver, $D$ denotes the diffusion coefficient of the information particles, and $r$ is the radius of the receiver that is assumed to have a spherical shape.

For ease of illustration, an On-Off Keying (OOK) modulation scheme is considered. At the $i$ th time-slot, the transmitter releases $N_{T X}$ information particles into the environment if the symbol is $s_{i}=1$ and no information particles if the symbol is $s_{i}=0$. Under these assumptions, the hitting probability of an absorbing receiver after $t$ seconds that an information particle is released can be expressed as follows:

$$
P_{h i t}(t)=\int_{0}^{t} f_{h i t}(t) d t
$$

From (1) and (2), we have:

$$
P_{\text {hit }}^{3 D}(t)=\int_{0}^{t} f_{\text {hit }}^{3 D}(t) d t=\frac{r}{d} \operatorname{erfc}\left(\frac{d-r}{\sqrt{4 D t}}\right)
$$

where $\operatorname{erf}(y)=\int_{0}^{y} \frac{2}{\sqrt{\pi}} e^{-x^{2}} d x$ and $\operatorname{erfc}(y)=1-\operatorname{erf}(y)$.

During the $(i-1)$ th time-slot, the probability that one information particle hits the receiver is:

$$
P_{i-1}=\int_{(i-1) T}^{i T} f_{h i t}(t) d t
$$

where $T$ is the duration of the time-slot, and we assume that the system is well synchronized such that there is no time offset.

From (1) and (4), we obtain:

$$
P_{i-1}^{3 D}=\frac{r}{d}\left\{\operatorname{erfc}\left(\frac{d-r}{\sqrt{4 D i T}}\right)-\operatorname{erfc}\left(\frac{d-r}{\sqrt{4 D(i-1) T}}\right)\right\} \text {. }
$$

Let $C_{j}=N_{T X} P_{j}$ denote the average number of received information particles at the $j$ th time-slot, where $P_{j}=P_{j}^{3 D}$ for ease of notation. The number of received information particles at the $i$ th time-slot follows a Poisson distribution [8]:

$$
r_{i} \sim \operatorname{Poisson}\left(I_{i}+s_{i} C_{0}\right)
$$

where $I_{i}=\lambda_{0} T+\sum_{j=1}^{\infty} s_{i-j} C_{j}$ is the sum of ISI and noise power, where $\lambda_{0}$ is the background noise power per unit time. More precisely, the probability of having $r_{i}$ information particles is:

$$
P\left(r_{i} \mid I_{i}+s_{i} C_{0}\right)=\frac{e^{-\left(I_{i}+s_{i} C_{0}\right)}\left(I_{i}+s_{i} C_{0}\right)^{r_{i}}}{r_{i} !}
$$

For ease of tractability, we assume that $C_{i}$ for $i>L$ is small enough to be included into the background noise. In this case, $L$ denotes the length of the Poisson channel. The Signal-to-Noise Ratio (SNR) is defined as follows:

$$
S N R=10 \log _{10} \frac{C_{0}}{2 \lambda_{0} T}
$$

where it is assumed that the information bits are equiprobable.

Accordingly, given the SNR, the number of released particles, $N_{T X}$, is as follows:

$$
N_{T X}=10^{\frac{S N R}{10}} 2 \lambda_{0} T
$$

For reference, the system parameters are listed in Table I. 
TABLE I

SYSTEM PARAMETERS

\begin{tabular}{|l|l|}
\hline \multicolumn{1}{|c|}{ Parameters } & \multicolumn{1}{c|}{ Value } \\
\hline$\lambda_{0}$ & $100 \mathrm{~s}^{-1}$ \\
\hline Receiver radius (r) & $45 \mathrm{~nm}$ \\
\hline Distance (d) & $500 \mathrm{~nm}$ \\
\hline Diffusion coefficient (D) & $4.265 \cdot 10^{-10} \mathrm{~m}^{2} / \mathrm{s}$ \\
\hline Discrete time length $\Delta T$ & $9 \mathrm{us}$ \\
\hline Time-slot duration (T) & $30 \Delta T, 50 \Delta T$ \\
\hline Channel length (L) & 6 \\
\hline
\end{tabular}

\section{Threshold-BASED DEMODUlation}

We study a threshold-based demodulator and denote by $\tau$ the demodulation threshold. Let $\bar{s}_{i}$ be the estimate of symbol $s_{i}$ at time-slot $i$, a threshold-based demodulator operates as:

$$
\bar{s}_{i}=\left\{\begin{array}{ll}
0, & r_{i} \leq \tau \\
1, & r_{i}>\tau
\end{array} .\right.
$$

In the following two sections, we optimize the detection threshold $\tau$ by relaying on a model-based and a data-driven approach, respectively.

\section{A. Model-Based Analysis and Optimization}

Let us consider the $i$ th time-slot and let $s_{i-1}=$ $\left\{s_{i-1}, s_{i-2}, \ldots s_{i-L}\right\}$ be the vector of bits received in the previous $L$ time-slots. The error probability can be written as follows:

$$
\begin{aligned}
P_{e}\left(s_{i-1}, \tau\right)= & \frac{1}{2}\left[P\left(r_{i} \geq \tau \mid s_{i}=0, s_{i-1}\right)\right. \\
& \left.+P\left(r_{i}<\tau \mid s_{i}=1, s_{i-1}\right)\right],
\end{aligned}
$$

where

$$
\begin{aligned}
& P\left(r_{i} \geq \tau \mid s_{i}=0, s_{i-1}\right)=P\left(r_{i} \geq \tau \mid \lambda_{0} T+\sum_{j=1}^{L} s_{i-j} C_{j}\right) \\
& =\sum_{k=\lceil\tau\rceil}^{\infty} \frac{e^{-\left(\lambda_{0} T+\sum_{j=1}^{L} s_{i-j} C_{j}\right)}\left(\lambda_{0} T+\sum_{j=1}^{L} s_{i-j} C_{j}\right)^{k}}{k !} \\
& =Q\left(\lambda_{0} T+\sum_{j=1}^{L} s_{i-j} C_{j},\lceil\tau\rceil\right),
\end{aligned}
$$

where $Q(\lambda, n)=\sum_{k=n}^{\infty} \frac{e^{-\lambda} \lambda^{k}}{k !}$ is the incomplete Gamma function and $Q(\lambda, 0)=1$. Similarly, we have:

$$
\begin{aligned}
& P\left(r_{i}<\tau \mid s_{i}=1, s_{i-1}\right)=P\left(r_{i}<\tau \mid \lambda_{0} T+\sum_{j=1}^{L} s_{i-j} C_{j}+C_{0}\right) \\
& =\sum_{k=0}^{\lceil\tau\rceil-1} \frac{e^{-\left(\lambda_{0} T+\sum_{j=1}^{L} s_{i-j} C_{j}+C_{0}\right)}\left(\lambda_{0} T+\sum_{j=1}^{L} s_{i-j} C_{j}+C_{0}\right)^{k}}{k !} \\
& =1-\sum_{k=\lceil\tau\rceil}^{\infty} \frac{e^{-\left(\lambda_{0} T+\sum_{j=1}^{L} s_{i-j} C_{j}+C_{0}\right)}\left(\lambda_{0} T+\sum_{j=1}^{L} s_{i-j} C_{j}+C_{0}\right)^{k}}{k !} \\
& =1-Q\left(\lambda_{0} T+\sum_{j=1}^{L} s_{i-j} C_{j}+C_{0},\lceil\tau\rceil\right)
\end{aligned}
$$

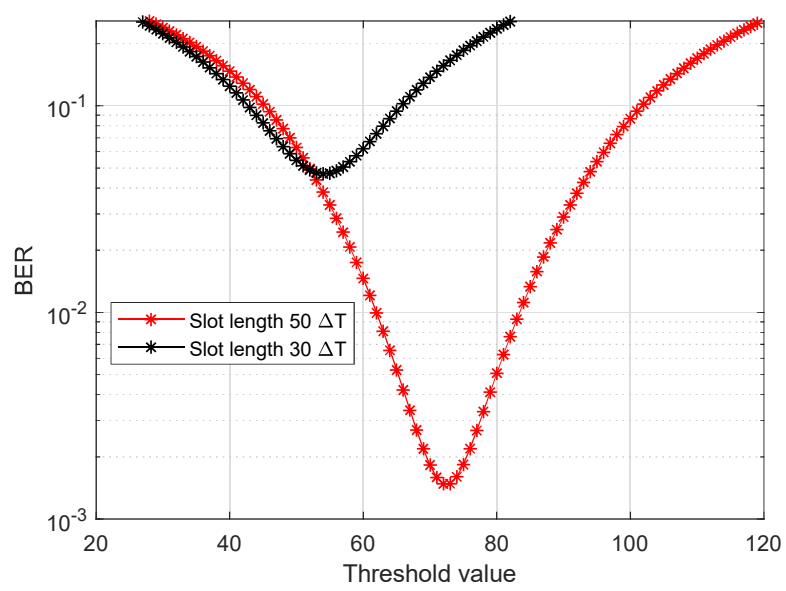

Fig. 2. Error probability in (14) as a function of $\tau$ (the SNR is $30 \mathrm{~dB}$ ).

From (11) and (12), we obtain:

$$
\begin{aligned}
P_{e}\left(s_{i-1}, \tau\right) & =\frac{1}{2}\left[Q\left(\lambda_{0} T+\sum_{j=1}^{L} s_{i-j} C_{j},\lceil\tau\rceil\right)\right. \\
& \left.+1-Q\left(\lambda_{0} T+\sum_{j=1}^{L} s_{i-j} C_{j}+C_{0},\lceil\tau\rceil\right)\right]
\end{aligned}
$$

Finally, the error probability can be obtained by averaging (13) with respect to the vector $s_{i-1}=\left\{s_{i-1}, s_{i-2}, \ldots s_{i-L}\right\}$, as follows:

$$
P_{e}(\tau)=\frac{1}{2^{L}} \sum_{s_{i-1}} P_{e}\left(s_{i-1}, \tau\right)
$$

In order to obtain appropriate performance and, thus, reduce the error probability in (14), the detection threshold, $\tau$, needs to be appropriately chosen and optimized. In Fig. 2, we depict (14) as a function of $\tau$. We observe that an optimal value of $\tau$ exists that minimizes the error probability and that depends on the time slot duration $T$, i.e., the amount of ISI.

In mathematical terms, the optimal threshold that minimizes the error probability can be formulated as follows:

$$
\tau^{*}=\underset{\tau}{\arg \min } \quad P_{e}(\tau)
$$

Due to the analytical complexity of (14), it is not possible to compute $\tau^{*}$ explicitly.

\section{B. Data-Driven Optimization}

In this section, we consider a data-driven approach where an ANN is trained based on raw data. The objective of the ANN is to demodulate the transmitted data by minimizing the error probability. More precisely, an ANN-based demodulator is a system whose input is the received information particles, $r_{i}$ at the $i$ th time-slot, and the outputs are the probabilities that the transmitted bit is 0 or 1, i.e., $P_{i}\left(s_{i}=0 \mid r_{i}\right)$ and $P_{i}\left(s_{i}=1 \mid r_{i}\right)$, respectively. Since, $P_{i}\left(s_{i}=1 \mid r_{i}\right)+P_{i}\left(s_{i}=0 \mid r_{i}\right)=1$, only one of the two probabilities is needed. In the sequel, we use 
TABLE II

PARAMETERS OF THE ANN

\begin{tabular}{|l|l|}
\hline Parameters & Value \\
\hline Number of layers & $\{1,10\}$ \\
\hline Learning rate & 0.01 \\
\hline Training epoch & 200 \\
\hline Replication times & 50 \\
\hline Number of training bits & 1000 \\
\hline Number of validation bits & 100000 \\
\hline
\end{tabular}

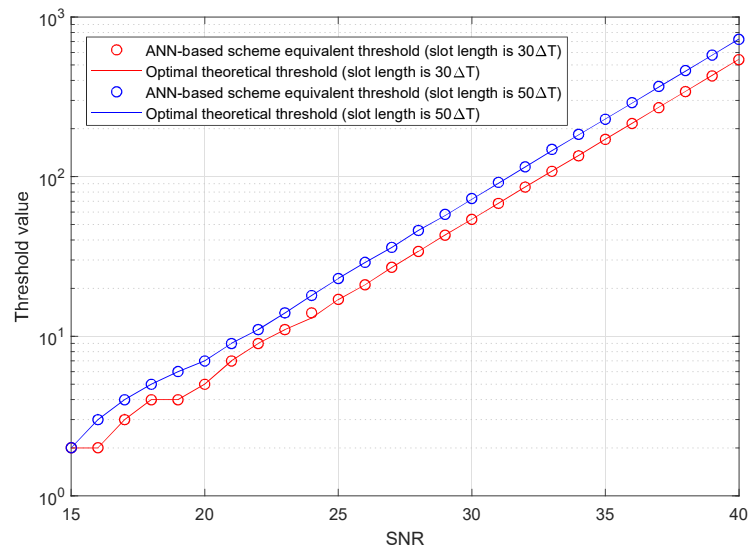

Fig. 3. Demodulation thresholds of model-based and data-driven (ANN) scheme with 10 layers.

the notation $P_{i}=P_{i}\left(s_{i}=1 \mid r_{i}\right)$. Based on the outputs, the ANN demodulate the received bits as follows:

$$
\bar{s}_{i}=\left\{\begin{array}{ll}
0, & P_{i} \leq 0.5 \\
1, & P_{i}>0.5
\end{array},\right.
$$

where the threshold 0.5 accounts for the fact that the bits are equiprobable.

In order to train the $\mathrm{ANN}$, we consider a supervised learning approach, i.e., we compute the parameters (e.g., the bias factors and the weights) of the ANN by using a known sequence of transmitted bits. In particular, we use the Bayesian regularization back propagation technique (trainbr), which updates the weights and biases by using the LevenbergMarquardt optimization algorithm. The set of parameters to train and operate the ANN are summarized in Table II. The training is performed in a batch mode, and, in particular, the replication time denotes the number of batches each of which is 1000 -bit long.

\section{NuMERICAL RESUlTS}

In Fig. 3, we compare the optimal threshold computed numerically from (15) as a function of the SNR, and the demodulation threshold that is learned by the ANN-based demodulator. In the latter case, the threshold is obtained, after completing the training of the $\mathrm{ANN}$, and identifying the input, i.e., the number of information particles, for which the output probability is equal to 0.5 . We observe that the ANNbased implementation is capable of learning the demodulation threshold in a very accurate manner.

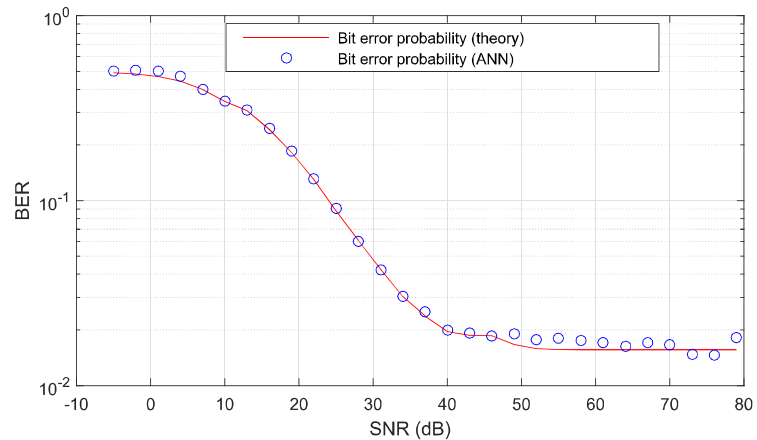

Fig. 4. Bit error probability of the ANN-based demodulator (10 layers) vs. the analytical framework in (14) $-T=30 \Delta T$.

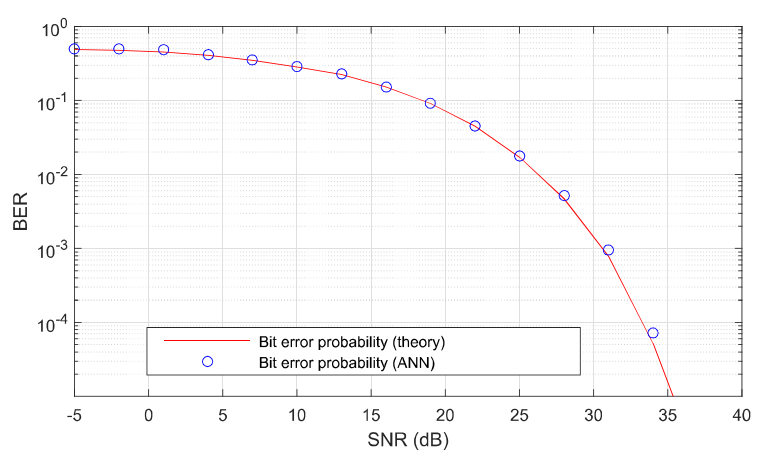

Fig. 5. Bit error probability of the ANN-based demodulator (10 layers) vs. the analytical framework in (14) $-T=50 \Delta T$.

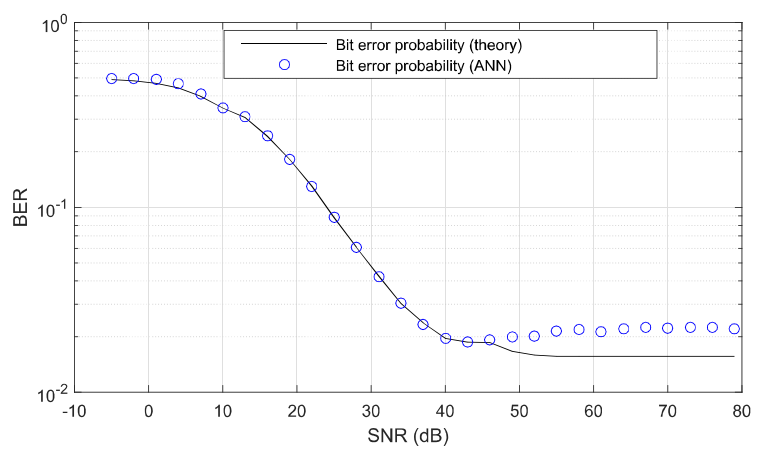

Fig. 6. Bit error probability of the ANN-based demodulator (1 layer) vs. the analytical framework in (14) $-T=30 \Delta T$.

In Fig. 4 and Fig. 5, we compare the bit error probability of the ANN-based demodulator with 10 layers against (14) by considering a short symbol time (small ISI) and a long symbol time (large ISI), respectively. As for the analytical model, the optimal threshold is estimated from (15) for each value of SNR. We note a very good agreement even with only 10 layers.

In Fig. 6 and Fig. 7, we conduct a similar study as for Fig. 4 and Fig. 5, but the ANN-based demodulator employs a single layer. In this case, the ANN-based demodulator is not capable 


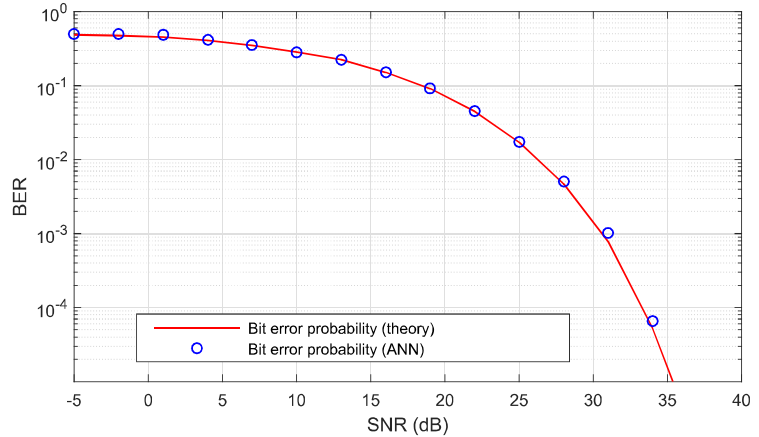

Fig. 7. Bit error probability of the ANN-based demodulator (1 layer) vs. the analytical framework in (14) $-T=50 \Delta T$

of perfectly following the analytical framework if the ISI is not negligible (as in Fig. 6).

\section{CONCLUSION}

In this paper, we have analyzed the potential of ANNs in order to obtain robust receiver schemes for application to $\mathrm{MC}$ systems. We have developed an exact analytical framework for the MC system that relies on the perfect knowledge of the channel model. Based on it, we have optimized the demodulation threshold in order to minimize the error probability. By using known learning methods and by training an ANN by using only raw data, we have shown that receivers that perform as well as those optimized by exploiting the exact knowledge of the system model can be obtained.

\section{REFERENCES}

[1] S. Hiyama, Y. Moritani, T. Suda, R. Egashira, A. Enomoto, M. Moore, and T. Nakano, Molecular Communication, 2005.

2] N. Farsad, H. B. Yilmaz, A. Eckford, C.-B. Chae, and W. Guo, "A comprehensive survey of recent advancements in molecular communication," IEEE Communications Surveys \& Tutorials, vol. 18, no. 3, pp 1887-1919, 2016.

[3] S. M. Rouzegar and U. Spagnolini, "Channel estimation for diffusive mimo molecular communications," in 2017 European Conference on Networks and Communications. IEEE, 2017, pp. 1-5.

[4] V. Jamali, A. Ahmadzadeh, C. Jardin, H. Sticht, and R. Schober, "Channel estimation for diffusive molecular communications," IEEE Transactions on Communications, vol. 64, no. 10, pp. 4238-4252, 2016.

[5] A. Noel, K. C. Cheung, and R. Schober, "Improving receiver performance of diffusive molecular communication with enzymes," IEEE Transactions on Nanobioscience, vol. 13, no. 1, pp. 31-43, 2014.

[6] M. Damrath and P. A. Hoeher, "Low-complexity adaptive threshold detection for molecular communication," IEEE Transactions on Nanobioscience, vol. 15, no. 3, pp. 200-208, 2016.

[7] D. Kilinc and O. B. Akan, "Receiver design for molecular communication," IEEE Journal on Selected Areas in Communications, vol. 31, no. 12, pp. 705-714, 2013.

[8] R. Mosayebi, H. Arjmandi, A. Gohari, M. Nasiri-Kenari, and U. Mitra "Receivers for diffusion-based molecular communication: Exploiting memory and sampling rate," IEEE Journal on Selected Areas in Communications, vol. 32, no. 12, pp. 2368-2380, 2014.

[9] V. Jamali, N. Farsad, R. Schober, and A. Goldsmith, "Non-coherent detection for diffusive molecular communication systems," IEEE Transactions on Communications, to appear, 2018.

[10] N. Farsad and A. Goldsmith, "Sliding bidirectional recurrent neural networks for sequence detection in communication systems," in IEEE International Conference on Acoustics, Speech and Signal Processing, 2018.
[11] Y. Deng, A. Noel, W. Guo, A. Nallanathan, and M. Elkashlan, "Analyzing large-scale multiuser molecular communication via 3-d stochastic geometry," IEEE Transactions on Molecular, Biological and Multi-Scale Communications, vol. 3, no. 2, pp. 118-133, 2017. 